\title{
Oscillator strengths and effective collision strengths for electron excitation of $\mathrm{Mg} \mathrm{VI^{ \star }}$
}

\begin{abstract}
S. S. Tayal
Department of Physics, Clark Atlanta University, Atlanta, GA 30314, USA

e-mail: stayal@cau.edu

Received 6 June 2012 / Accepted 2 October 2012

ABSTRACT

Aims. Electron impact excitation collision strengths and oscillator strengths for the astrophysically important lines in Mg VI are reported. Thermally averaged collision strengths are presented as a function of electron temperature for application to solar and other astrophysical plasmas.

Methods. The collision strengths were calculated in a close-coupling approximation using the B-spline Breit-Pauli R-matrix method. The multiconfiguration Hartree-Fock method with term-dependent, non-orthogonal orbitals was employed for an accurate representation of the target wave functions. The close-coupling expansion includes 74 bound levels of $\mathrm{Mg}$ VI covering the $n=2$ and $n=3$ terms. The present calculations led to a total of 2701 forbidden, intercombination, and allowed transitions between fine-structure levels. The effective collision strengths were obtained by averaging the electron collision strengths over a Maxwellian distribution of velocities. Results. The accuracy of present oscillator strengths is evaluated by the agreement between the length and velocity formulations combined with the agreement between the calculated and measured excitation energies. The calculated excitation energies are in excellent agreement with experiments and other extensive configuration-interaction calculations. The oscillator strengths for all E1 transitions are listed. The effective collision strengths are tabulated for all 2701 transitions among the 74 fine-structure levels at 11 electron temperatures in the range from 10000 to $200000 \mathrm{~K}$. The present results are compared with other close-coupling and distorted-wave calculations. Overall good agrement is generally found with the calculations by Ramsbottom \& Bell and by Landi \& Bhatia for many transitions, but significant differences are also noted for some transitions.
\end{abstract}

Key words. atomic data - atomic processes - line: formation

\section{Introduction}

The prominent Mg VI emission lines have been observed in the ultraviolet (UV), extreme ultraviolet (EUV), and soft X-ray spectral ranges in solar and various other astrophysical plasmas. The intensity maps for Mg VI and Fe VII ions are similar, indicating a similar temperature of formation. The maximum ionic abundance of $\mathrm{Mg}$ VI in ionization equilibrium of astrophysical plasmas is at around $4 \times 10^{5} \mathrm{~K}$. The Mg VI lines due to the $2 \mathrm{~s}^{2} 2 \mathrm{p}^{3}{ }^{2} \mathrm{D}_{3 / 2,5 / 2}^{\circ}-2 \mathrm{~s} 2 \mathrm{p}^{4}{ }^{2} \mathrm{P}_{1 / 2,3 / 2}, 2 \mathrm{~s} 2 \mathrm{p}^{4}{ }^{2} \mathrm{D}_{5 / 2,3 / 2}$ transitions at 268.99 and 270.40 have been observed with Hinode/EIS (Young et al. 2007; Brooks et al. 2009) and with SOHO/CDS at transition region temperatures below $10^{6} \mathrm{~K}$. The Mg VI forbidden lines are identified at 1190.09 and $1191.64 \AA$ in the spectra of solar corona by SOHO/SUMER instrument. Several lines of $\mathrm{N}$-like Mg VI due to the $2 \mathrm{~s}^{2} 2 \mathrm{p}^{3}-2 \mathrm{~s} 2 \mathrm{p}^{4}$ transitions have been observed with SERTS including lines at 349.11 and $349.18 \AA$ in the EUV spectrum of solar active region (Thomas \& Neupert 1994). The Mg VI lines have also been observed in the spectrum of symboitic stars AG Dra and RR Tel by Hubble/STIS instrument (Young et al. 2006).

The N-like ions can provide some of the best density diagnostics of solar transition region in both quiet and active conditions using intensity ratios from $n=2$ forbidden and allowed lines. The forbidden lines among the ground configuration occur

* Tables 3 and 4 are available at the CDS via anonymous ftp to cdsarc.u-strasbg.fr $(130.79 .128 .5)$ or via

http://cdsarc.u-strasbg.fr/viz-bin/qcat?J/A+A/548/A27 in UV spectral region and the allowed lines among the $n=2$ levels occur in the EUV spectral region. The Mg VI line intensity ratios between the $n=2$ and $n=3$ levels are temperature sensitive and can be used as plasma temperature diagnostics over a wide temperature range of the solar corona. There are inconsistencies between the observed and theoretical predictions for line intensities of N-like Mg VI and Si VIII ions due to the atomic data for these ions (Bhatia \& Landi 2003; Young et al. 1998). For example, there are discrepancies between theory and observations from the SERTS for the Mg VI $2 s^{2} 2 p^{3}{ }^{2} D^{\circ}-2 s 2 p^{4}{ }^{2} P,{ }^{2} D$ and $2 s^{2} 2 p^{3}{ }^{4} S^{\circ}-2 s 2 p^{4}{ }^{4} P$ lines which have been attributed to the inaccuracies in the atomic data (Young et al. 1998). In order to make full use of high quality EIS spectroscopic measurements, accurate atomic data of matching quality are needed (Young \& Landi 2009; Brooks et al. 2009). Radiative transitions and electron-ion collisions play an important role in the understanding of the physical processes and conditions in various types of astrophysical plasmas including solar transition region in both quiet and active conditions.

Several theoretical calculations of collision strengths and rates have been performed using distorted-wave and closecoupling methods with different approximations for the target wave functions and with different number of target states. To our knowledge, there is no experimental study available for electron impact excitation collision data of Mg VI. The experimental data are important to verify or to calibrate theory. In astrophysical plasma applications, however, the majority of required radiative decay rates and collisional rate coefficients are derived from the 
calculations. Previous theoretical calculations for electron impact excitation collision strengths include the distorted-wave calculations of Bhatia \& Mason (1980), Bhatia \& Young (1998), Landi \& Bhatia (2007), and Zhang \& Sampson (1999) and in the R-matrix calculations of Ramsbottom \& Bell (1997). The distorted-wave calculations were carried out at a few selected incident electron energies and did not include resonances in the collision strengths. Bhatia \& Young (1998) and Ramsbottom \& Bell (1997) considered the 12 lowest LS states of the $2 s^{2} 2 p^{3}$, $2 \mathrm{~s} 2 \mathrm{p}^{4}, 2 \mathrm{p}^{5}$, and $2 \mathrm{~s}^{2} 2 \mathrm{p}^{2} 3 \mathrm{~s}$ configurations, while Landi \& Bhatia (2007) included 74 fine-structure levels of the $n=2$ and $n=$ 3 states in their calculations.

Previous theoretical calculations have basically been performed in the LS-coupling approximation and the collision strengths for fine-structure transitions were determined by transforming scattering matrices in LS coupling to LSJ coupling (Saraph 1978). Besides these, the close-coupling expansions did not include all possible $n=3$ terms which may be important for the convergence of the close-coupling expansion. Ramsbottom $\&$ Bell (1997) included only the four $2 s^{2} 2 p^{2} 3 s$ states of the $n=3$ complex in their 12-state R-matrix calculation. The purpose of the work reported here is to provide a set of accurate oscillator strengths and effective collision strengths for electron excitation of Mg VI for applications to astrophysical plasmas. Our scattering calculations were performed by using highly accurate target wave functions and by including fine-structure effects in the close-coupling expansions directly to make sure that the relativistic effects are adequately accounted for in the scattering calculations. The present calculations were carried out with the $B$-spline Breit-Pauli $R$-matrix (BSR) approach (Zatsarinny \& Tayal 2001; Zatsarinny 2006). One of the distinct features of the present method is the use of term-dependent, non-orthogonal orbital sets in the description of the target states. This allows us to optimize the atomic wave functions for different states independently, resulting in a more accurate target description than those used in previous collision calculations.

\section{Computational methods}

We used non-orthogonal orbitals to represent different $\mathrm{Mg}$ VI states. The non-orthogonal orbitals provide much greater flexibility in the choice of wave functions than the orthogonal orbitals and also allow us to include correlation with a reasonable number of configurations and correlated orbitals. Our calculations were performed using the multiconfiguration Hartree-Fock (MCHF) method (Froese Fischer 2007; Zatsarinny $\&$ Froese Fischer 2000). In the MCHF approach the atomic state is represented by an atomic state function

$\Psi(\alpha \mathrm{LS})=\sum_{i} c_{i} \Phi\left(\alpha_{i} \mathrm{LS}\right)$

where the configuration state functions (CSF) $\Phi\left(\alpha_{i} \mathrm{LS}\right)$ are constructed from one-electron functions and $\alpha_{i}$ defines the coupling of angular momenta of the electrons.

The Mg VI wave functions exhibit significant correlation corrections and term dependence of the one-electron orbitals. The non-orthogonal orbitals were optimized for each atomic state separately. We began with the Hartee-Fock (HF) calculation for the $1 \mathrm{~s}, 2 \mathrm{~s}$, and $2 \mathrm{p}$ orbitals for the ground $2 \mathrm{~s}^{2} 2 \mathrm{p}^{3}$ configuration terms and then determined separate sets of excited orbitals for various symmetries of even and odd parities. A set of correlation s, p, d, f, and $\mathrm{g}$ orbitals was determined by optimization on the ground $2 \mathrm{~s}^{2} 2 \mathrm{p}^{3}{ }^{4} \mathrm{~S}^{\circ}$ state and another set of $\mathrm{s}, \mathrm{p}, \mathrm{d}, \mathrm{f}$, and $\mathrm{g}$ correlation orbitals was optimized on the $2 s^{2} 2 p^{2} 3 s^{4} \mathrm{P}$ state. The mean radii of the correlation orbitals are comparable to the spectroscopic orbitals and thus the correlation corrections are represented very well. The spectroscopic and correlation functions are used to construct CI expansions for different atomic states by allowing one-electron and twoelectron excitations from all the basic configurations $2 \mathrm{~s}^{2} 2 \mathrm{p}^{3}$, $2 s 2 p^{4}, 2 s^{2} 2 p^{2} 3 s, 2 s^{2} 2 p^{2} 3 p$, and $2 s^{2} 2 p^{2} 3 d$ to the spectroscopic and correlation orbitals used in our calculations. In the construction of CI expansions for fine-structure levels with various $\mathrm{J}$ and $\pi$, we used configurations generated in this excitation scheme for the atomic LS states and with insignificant configurations with coefficients less than 0.0008 omitted from the expansions. We retained 3314 and 2267 configurations for even and odd parity levels, respectively, in our calculations of oscillator strengths.

We further omitted configurations with weights less than 0.01 in calculations for the description of target wave functions to make the subsequent scattering calculations computationally feasible. We included 226 and 272 configuration state functions in the CI expansions of levels of odd parity and even parity respectively in our final calculation. We used 20, 69, 48, $13,29,17$, and $30 \mathrm{CSF}$ respectively for the representation of the ${ }^{2} \mathrm{~S}^{\circ},{ }^{2} \mathrm{P}^{\circ},{ }^{2} \mathrm{D}^{\circ},{ }^{2} \mathrm{~F}^{\circ},{ }^{4} \mathrm{~S}^{\circ},{ }^{4} \mathrm{P}^{\circ}$, and ${ }^{4} \mathrm{D}^{\circ}$ terms of odd parity. For the ${ }^{2} \mathrm{~S},{ }^{2} \mathrm{P},{ }^{2} \mathrm{D},{ }^{2} \mathrm{~F},{ }^{2} \mathrm{G},{ }^{4} \mathrm{P},{ }^{4} \mathrm{D}$, and ${ }^{4} \mathrm{~F}$ even parity terms we used $31,74,51,33,10,44,15$, and 14 CSFs respectively in the $\mathrm{CI}$ expansions. The valence-shell correlation effects due to electron excitations to the $2 p^{4} 3 s, 2 p^{4} 3 p$, and $2 p^{4} 3 d$ configurations are essential for the accurate representation of target states. The 2 s electron excitation from the $2 \mathrm{~s}^{2} 2 \mathrm{p}^{3}$ ground configuration to the $3 \mathrm{~s}, 3 \mathrm{p}$, and $3 \mathrm{~d}$ spectroscopic orbitals is very important. The configurations with one-electron virtual excitations to the correlation $\mathrm{s}, \mathrm{p}$, and $\mathrm{d}$ orbitals are also important. Our calculation takes the important correlation and relaxation effects and of the term dependence of the valence orbitals into account.

For the scattering calculations we employed the BSR code (Zatsarinny 2006). Details of this approach can be found in our recent publications on e-K II (Tayal \& Zatsarinny 2008) and e-S II (Tayal \& Zatsarinny 2010). The distinctive feature of the method is the use of B-splines as a universal basis to represent the scattering orbitals in the inner region of $r \leq a$. Hence, the $\mathrm{R}$-matrix expansion in this region takes the form

$$
\begin{aligned}
\Psi_{k}\left(x_{1}, \ldots, x_{N+1}\right) & =\mathcal{A} \sum_{i j} \bar{\Phi}_{i}\left(x_{1}, \ldots, x_{N} ; \hat{\boldsymbol{r}}_{N+1} \sigma_{N+1}\right) \\
\times & r_{N+1}^{-1} B_{j}\left(r_{N+1}\right) a_{i j k}+\sum_{i} \chi_{i}\left(x_{1}, \ldots, x_{N+1}\right) b_{i k} .
\end{aligned}
$$

Here $\bar{\Phi}_{i}$ are the channel functions while the splines $B_{j}(r)$ represent the continuum orbitals. The $\chi_{i}$ are $(N+1)$-electron configurations formed from the one-electron orbitals used to describe the $N$-electron target states. These must be included to ensure completeness of the total trial wave function if the continuum orbitals are constructed orthogonally to the bound orbitals. The expansion coefficients $a_{i j k}$ and $b_{i k}$ are determined by diagonalizing the $(N+1)$-electron Breit-Pauli Hamiltonian with added Bloch operator. The relativistic effects in the scattering calculations have been incorporated in the Breit-Pauli Hamiltonian through the use of Darwin, mass correction, and spin-orbit operators.

The present scattering approach imposes only limited orthogonality conditions to the continuum orbitals. The use of nonorthogonal orbitals allows us to reduce or even to avoid the introduction of additional $(N+1)$-electron terms in the R-matrix expansion. We only require the orthogonality of continuum 
orbitals to the bound orbitals in the filled 1s shell. No orthogonality constraints to the spectroscopic excited orbitals or the correlated orbitals were imposed. In order to represent the continuum orbitals in internal region, $48 \mathrm{~B}$-splines of order 8 were used in the present calculations, with the size of the B-spline box $a=7.25 a_{0}\left(a_{0}=0.529 \times 10^{-10} \mathrm{~m}\right.$ denoting the Bohr radius $)$. The scattering parameters are found by matching the inner solution at $r=a$ with asymptotic solutions in the outer region. The Seaton's STGF program as extended by Badnell (1999) has been employed to find the asymptotic solutions and collision parameters. We numerically calculated partial-wave contributions up to $J=30$. These partial waves are sufficient to achieve convergence for the forbidden transitions under consideration. Higher partial wave contributions are needed for the dipole-allowed transitions and these are estimated in a top-up procedure based on the Coulomb-Bethe method (Burgess \& Sheorey 1974) or on geometric series approximation. In order to resolve the resonance structure with high accuracy, we used a fine energy mesh of $2.0 \times$ $10^{-4} \mathrm{Ry}$ in the closed-channel energy region up to $6.0 \mathrm{Ry}$ and then an energy mesh of $1.0 \times 10^{-3}$ Ry up to $10.0 \mathrm{Ry}$, that allowed us to resolve a vast majority of the narrow resonances in the energy range up through the highest excitation threshold. At higher energies up to 70 Ry where all channels are open and there are no resonances, the cross sections show smooth behavior and we used an energy grid of $0.25 \mathrm{Ry}$.

In astrophysical applications it is convenient to use excitation rate coefficients or thermally averaged collision strengths as a function of electron temperature. The excitation rates are obtained by averaging collision strengths over a Maxwellian distribution of electron energies. The excitation rate coefficient for a transition from state $i$ to state $f$ at electron temperature $T_{\mathrm{e}}$ is given by

$C_{i f}=\frac{8.629 \times 10^{-6}}{g_{i} T_{\mathrm{e}}^{1 / 2}} \gamma_{i f}\left(T_{\mathrm{e}}\right) \exp \left(\frac{-\Delta E_{i f}}{k T_{\mathrm{e}}}\right) \mathrm{cm}^{3} \mathrm{~s}^{-1}$,

where $g_{i}$ is the statistical weight of the lower level $i, \Delta E_{i f}=$ $E_{f}-E_{i}$ is the excitation energy and $\gamma_{i f}$ is a dimensionless quantity called effective collision strength given by

$\gamma_{i f}\left(T_{\mathrm{e}}\right)=\int_{0}^{\infty} \Omega_{i f} \exp \left(\frac{-E_{f}}{k T_{\mathrm{e}}}\right) \mathrm{d}\left(\frac{E_{f}}{k T_{\mathrm{e}}}\right)$,

where $E_{f}$ is the energy of incident electron with respect to the upper level $f$. If the collision strength is assumed to be independent of the incident electron energy, we have $\gamma_{i f}=\Omega_{i f}$. The effective collision strengths are calculated by integrating collision strengths for fine-structure levels over a Maxwellian distribution of electron energies. The integration in Eq. (4) should be carried out using energy dependent collision strengths from threshold to infinity. The collision strengths vary smoothly in high energy region and the energy dependence of collision strengths for high energies have been properly accounted for by using numerical extrapolation technique at $E>70 \mathrm{Ry}$. In the asymptotic region, the collision strengths follow a high energy limiting behavior for the dipole-allowed transitions

$\Omega_{i f}(E) \sim_{E \rightarrow \infty} \frac{4 S}{3} \ln (E)$,

where $S$ is the line strength. The collision strengths vary smoothly in the high energy region and exhibit an increasing trend for dipole-allowed transitions. The collision strength increases more rapidly for the stronger dipole-allowed transitions than the weaker transitions.

\section{Results and discussion}

The target states included in the present scattering calculations are given in Table 1, where we compare our calculated excitation energies with the available experimental values and with recent calculations by Froese Fischer \& Tachiev (2004) and Landi $\&$ Bhatia (2007). The overall agreement between experiment and present calculation is very satisfactory with an average deviation of about $0.034 \mathrm{Ry}$. The present excitation energies obtained from wave functions used in the scattering calculation show an average deviations of $0.019,0.047,0.053$, and $0.017 \mathrm{Ry}$ for the levels of $2 s^{2} 2 p^{3}, 2 s 2 p^{4}, 2 p^{5}$, and $2 s^{2} 2 p^{2} 3 l$ configurations respectively. The accuracy of excitation energies from our extensive MCHF calculation for transition rates is somewhat better with average deviation of about $0.01 \mathrm{Ry}$. The present structure description represents a substantial improvement over those used in previous R-matrix calculations by Ramsbottom \& Bell (1997) and distorted-wave calculations by Landi \& Bhatia (2007). We obtained good agreement for all $2 \mathrm{~s}^{2} 2 \mathrm{p}^{3}, 2 \mathrm{~s} 2 \mathrm{p}^{4}, 2 \mathrm{p}^{5}$, and $2 \mathrm{~s}^{2} 2 \mathrm{p}^{2} 3 l$ states, indicating that we consistently take correlation corrections in the ground and excited states into account, as our method allows us to use non-orthogonal orbitals, that are specific for given configurations. The accuracy of the present calculations is comparable to the Breit-Pauli MCHF calculation by Froese Fischer \& Tachiev (2004).

We also present the lifetimes of excited levels in Table 1, where our results are compared with the results of Froese Fischer $\&$ Tachiev (2004). The present lifetimes are within $10 \%$ of the results of Froese Fischer \& Tachiev (2004) for most of the levels. However, larger differences are found for the levels of the $2 s^{2} 2 p^{2} 3 p^{2} S^{\circ},{ }^{4} S^{\circ}, 2 s^{2} 2 p^{2} 3 d^{4} D,{ }^{2} F$, and ${ }^{2} G$ terms. The present lifetimes were calculated from transition probabilities of all possible E1 transitions between the states considered in our calculation. However, we have not included transition probabilities for forbidden E2 and M1 transitions and, therefore, the lifetimes of the metastable $2 \mathrm{~s}^{2} 2 \mathrm{p}^{3}{ }^{2} \mathrm{D}^{\circ}$ and ${ }^{2} \mathrm{P}^{\circ}$ levels are not given in Table 1. This may also contribute to some of the discrepancies with the work of Froese Fischer \& Tachiev (2004), especially for levels which decay primarily via forbidden transitions. The lifetimes of the $2 s 2 p^{4}, 2 p^{5}$ and $2 s^{2} 2 p^{2} 3 l$ configurations varies from a few nanoseconds to fraction of nanosecond with significant lifetimes for the $2 s^{2} 2 p^{2} 3 p^{2} S^{\circ},{ }^{4} S^{\circ},{ }^{2} D^{\circ},{ }^{4} D^{\circ}$, and $2 s^{2} 2 p^{2} 3 d^{2} G$ levels.

The accuracy of oscillator strengths to some extent relates to the quality of target wave functions and, therefore, to the accuracy and reliability of collision strengths and rate coefficients. For example, collision strengths of the dipole-allowed transitions at high electron energies are directly proportional to the oscillator strengths. We have presented our length values of oscillator strengths and transition probabilities in Table 2 where present results are compared with the calculations of Froese Fischer \& Tachiev (2004) and Landi \& Bhatia (2007). We used experimental energies in the calculations of oscillator strengths and radiative rates. A rather reasonable agreement between different calculations is noted. Our calculation shows somewhat better agreement with the results of Froese Fischer \& Tachiev (2004). We present oscillator strengths and transition probabilities for allowed and intercombination lines between the 74 fine-structure levels in Table 3. The relativistic effects were included through the spin-orbit, spin-other-orbit, spin-spin, mass and Darwin Breit-Pauli operators. We have presented oscillator strengths in both length and velocity formulations and transition probabilities in length form in Table 3. We have also listed wavelengths in this table. The values of oscillator strengths for intercombination lines are usually much lower than those for the 
Table 1. Excitation energies (in Ry) of the 74 target levels included in the present transition rates and scattering calculations.

\begin{tabular}{|c|c|c|c|c|c|c|c|c|}
\hline \multirow[t]{2}{*}{ Index } & \multirow[t]{2}{*}{ State } & \multirow[t]{2}{*}{$2 \mathrm{~J}$} & \multicolumn{4}{|c|}{ Excitation energy } & \multicolumn{2}{|c|}{$\tau(\mathrm{ns})$} \\
\hline & & & Present & Experiment & LB & FT & Present & FT \\
\hline 1 & $2 s^{2} 2 p^{3}{ }^{4} s^{\circ}$ & 3 & 0.00000 & 0.00000 & 0.00000 & 0.00000 & & \\
\hline 2 & $2 \mathrm{~s}^{2} 2 \mathrm{p}^{3}{ }^{2} \mathrm{D}^{\circ}$ & 5 & 0.51559 & 0.50444 & 0.54930 & 0.50803 & & \\
\hline 3 & $2 \mathrm{~s}^{2} 2 \mathrm{p}^{3}{ }^{2} \mathrm{D}^{\circ}$ & 3 & 0.51583 & 0.50459 & 0.54922 & 0.50811 & & \\
\hline 4 & $2 \mathrm{~s}^{2} 2 \mathrm{p}^{3}{ }^{2} \mathrm{P}^{\circ}$ & 1 & 0.79168 & 0.76474 & 0.79683 & 0.76643 & & \\
\hline 5 & $2 \mathrm{~s}^{2} 2 \mathrm{p}^{32} \mathrm{P}^{\circ}$ & 3 & 0.79233 & 0.76572 & 0.79774 & 0.76740 & & \\
\hline 6 & $2 \mathrm{~s} 2 \mathrm{p}^{4}{ }^{4} \mathrm{P}$ & 5 & 2.26796 & 2.25947 & 2.30067 & 2.26550 & $3.51-01$ & $3.59-01$ \\
\hline 7 & $2 \mathrm{~s} 2 \mathrm{p}^{4}{ }^{4} \mathrm{P}$ & 3 & 2.28220 & 2.27438 & 2.31656 & 2.28034 & $3.44-01$ & $3.52-01$ \\
\hline 8 & $2 s 2 p^{4}{ }^{4} P$ & 1 & 2.29064 & 2.28227 & 2.32500 & 2.28814 & $3.40-01$ & $3.48-01$ \\
\hline 9 & $2 \mathrm{~s} 2 \mathrm{p}^{4}{ }^{2} \mathrm{D}$ & 5 & 3.17356 & 3.11426 & 3.24179 & 3.12598 & $1.30-01$ & $1.33-01$ \\
\hline 10 & $2 \mathrm{~s} 2 \mathrm{p}^{4}{ }^{2} \mathrm{D}$ & 3 & 3.17368 & 3.11465 & 3.24182 & 3.12632 & $1.29-01$ & $1.32-01$ \\
\hline 11 & $2 s 2 p^{42} s$ & 1 & 3.71777 & 3.66167 & 3.79323 & 3.67329 & $6.49-02$ & 6.79-02 \\
\hline 12 & $2 \mathrm{~s} 2 \mathrm{p}^{4}{ }^{2} \mathrm{P}$ & 3 & 3.94010 & 3.87462 & 4.04521 & 3.89437 & $2.96-02$ & $3.01-02$ \\
\hline 13 & $2 \mathrm{~s} 2 \mathrm{p}^{4}{ }^{2} \mathrm{P}$ & 1 & 3.95782 & 3.89234 & 4.06414 & 3.91202 & $2.96-02$ & $3.01-02$ \\
\hline 14 & $2 \mathrm{p}^{5}{ }^{5} \mathrm{P}^{\circ}$ & 3 & 5.99410 & 5.94025 & 6.19284 & 5.96734 & $5.31-02$ & $5.30-02$ \\
\hline 15 & $2 \mathrm{p}^{5}{ }^{2} \mathrm{P}^{\circ}$ & 1 & 6.01747 & 5.96400 & 6.21804 & 5.99090 & $5.27-02$ & $5.26-02$ \\
\hline 16 & $2 s^{2} 2 p^{2}\left({ }^{3} P\right) 3 s^{4} P$ & 1 & 8.12929 & 8.14620 & 8.08111 & 8.14209 & $4.67-02$ & $4.52-02$ \\
\hline 17 & $2 s^{2} 2 p^{2}\left({ }^{3} P\right) 3 s^{4} P$ & 3 & 8.13799 & 8.15480 & 8.09059 & 8.15137 & $4.66-02$ & $4.49-02$ \\
\hline 18 & $2 s^{2} 2 p^{2}\left({ }^{3} P\right) 3 s^{4} P$ & 5 & 8.15186 & 8.16900 & 8.10567 & 8.16514 & $4.66-02$ & $4.46-02$ \\
\hline 19 & $2 \mathrm{~s}^{2} 2 \mathrm{p}^{2}\left({ }^{3} \mathrm{P}\right) 3 \mathrm{~s}^{2} \mathrm{P}$ & 1 & 8.26274 & 8.27800 & 8.22972 & 8.27538 & $1.51-02$ & $1.53-02$ \\
\hline 20 & $2 s^{2} 2 p^{2}\left({ }^{3} P\right) 3 s^{2} P$ & 3 & 8.27964 & 8.29530 & 8.24783 & 8.29225 & $1.51-02$ & $1.53-02$ \\
\hline 21 & $2 s^{2} 2 p^{2}\left({ }^{1} D\right) 3 s^{2} D$ & 5 & 8.56657 & 8.55520 & 8.51646 & 8.55519 & $2.51-02$ & $2.46-02$ \\
\hline 22 & $2 s^{2} 2 p^{2}\left({ }^{1} D\right) 3 s^{2} D$ & 3 & 8.56684 & 8.55520 & 8.51663 & 8.55544 & $2.52-02$ & $2.46-02$ \\
\hline 23 & $2 s^{2} 2 p^{2}\left({ }^{3} P\right) 3 p^{2} S^{\circ}$ & 1 & 8.64961 & & 8.59376 & 8.64711 & $3.64+00$ & $4.57+00$ \\
\hline 24 & $2 s^{2} 2 p^{2}\left({ }^{3} P\right) 3 p^{4} D^{\circ}$ & 1 & 8.73632 & & 8.65723 & 8.71685 & $1.98+00$ & $2.16+00$ \\
\hline 25 & $2 s^{2} 2 p^{2}\left({ }^{3} P\right) 3 p^{4} D^{\circ}$ & 3 & 8.74132 & & 8.66253 & 8.72208 & $1.94+00$ & $2.11+00$ \\
\hline 26 & $2 s^{2} 2 p^{2}\left({ }^{3} P\right) 3 p^{4} D^{\circ}$ & 5 & 8.74910 & & 8.67154 & 8.73079 & $1.91+00$ & $2.10+00$ \\
\hline 27 & $2 s^{2} 2 p^{2}\left({ }^{3} P\right) 3 p^{4} D^{\circ}$ & 7 & 8.76219 & & 8.68435 & 8.74265 & $1.97+00$ & $2.14+00$ \\
\hline 28 & $2 s^{2} 2 p^{2}\left({ }^{3} P\right) 3 p{ }^{4} P^{\circ}$ & 1 & 8.77543 & & 8.70784 & 8.76836 & $4.54-01$ & $5.22-01$ \\
\hline 29 & $2 s^{2} 2 p^{2}\left({ }^{3} P\right) 3 p{ }^{4} P^{\circ}$ & 3 & 8.77937 & & 8.71199 & 8.77203 & $4.49-01$ & $5.16-01$ \\
\hline 30 & $2 s^{2} 2 p^{2}\left({ }^{3} P\right) 3 p{ }^{4} P^{\circ}$ & 5 & 8.78770 & & 8.72094 & 8.78038 & $4.52-01$ & 5.24-01 \\
\hline 31 & $2 s^{2} 2 p^{2}\left({ }^{3} P\right) 3 p{ }^{4} S^{\circ}$ & 3 & 8.88603 & & 8.82595 & 8.88461 & $2.73+00$ & $1.30-01$ \\
\hline 32 & $2 \mathrm{~s}^{2} 2 \mathrm{p}^{2}\left({ }^{3} \mathrm{P}\right) 3 \mathrm{p}^{2} \mathrm{D}^{\circ}$ & 3 & 8.88946 & & 8.80457 & 8.84789 & $2.61+00$ & $2.05+00$ \\
\hline 33 & $2 \mathrm{~s}^{2} 2 \mathrm{p}^{2}\left({ }^{3} \mathrm{P}\right) 3 \mathrm{p}^{2} \mathrm{D}^{\circ}$ & 5 & 8.90607 & & 8.82226 & 8.86497 & $2.49+00$ & $1.98+00$ \\
\hline 34 & $2 s^{2} 2 p^{2}\left({ }^{3} \mathrm{P}\right) 3 p^{2} \mathrm{P}^{\circ}$ & 1 & 8.96365 & & 8.89309 & 8.93579 & $4.60-01$ & $5.71-01$ \\
\hline 35 & $2 s^{2} 2 p^{2}\left({ }^{3} \mathrm{P}\right) 3 \mathrm{p}^{2} \mathrm{P}^{\circ}$ & 3 & 8.96511 & & 8.89557 & 8.93745 & $4.56-01$ & $5.66-01$ \\
\hline 36 & $2 s^{2} 2 p^{2}\left({ }^{1} S\right) 3 s^{2} S$ & 1 & 8.97406 & 8.96160 & & 8.96145 & $2.64-02$ & $2.55-02$ \\
\hline 37 & $2 s^{2} 2 p^{2}\left({ }^{1} D\right) 3 p^{2} F^{\circ}$ & 5 & 9.17886 & & 9.11154 & 9.15066 & $8.59-01$ & $9.83-01$ \\
\hline 38 & $2 s^{2} 2 p^{2}\left({ }^{1} D\right) 3 p^{2} F^{\circ}$ & 7 & 9.18247 & & 9.11521 & 9.15455 & $8.54-01$ & $9.77-01$ \\
\hline 39 & $2 \mathrm{~s}^{2} 2 \mathrm{p}^{2}\left({ }^{1} \mathrm{D}\right) 3 \mathrm{p}^{2} \mathrm{D}^{\circ}$ & 5 & 9.29677 & & 9.23722 & 9.24872 & $4.36-01$ & $5.92-01$ \\
\hline 40 & $2 s^{2} 2 p^{2}\left({ }^{1} D\right) 3 p^{2} D^{\circ}$ & 3 & 9.29868 & & 9.23825 & 9.25053 & 4.33-01 & 5.84-01 \\
\hline 41 & $2 \mathrm{~s}^{2} 2 \mathrm{p}^{2}\left({ }^{1} \mathrm{D}\right) 3 \mathrm{p}^{2} \mathrm{P}^{\circ}$ & 1 & 9.35653 & & 9.31797 & 9.33100 & $2.38-01$ & 2.74-01 \\
\hline 42 & $2 s^{2} 2 p^{2}\left({ }^{1} D\right) 3 p^{2} \mathrm{P}^{\circ}$ & 3 & 9.36626 & & 9.32836 & 9.33988 & $2.37-01$ & $2.73-01$ \\
\hline 43 & $2 s^{2} 2 p^{2}\left({ }^{3} P\right) 3 d^{4} F$ & 3 & 9.44021 & & 9.36532 & 9.41815 & $1.01+00$ & $1.05+00$ \\
\hline 44 & $2 \mathrm{~s}^{2} 2 \mathrm{p}^{2}\left({ }^{3} \mathrm{P}\right) 3 \mathrm{~d}^{4} \mathrm{~F}$ & 5 & 9.44476 & & 9.37042 & 9.42286 & $9.41-01$ & $9.88-01$ \\
\hline 45 & $2 s^{2} 2 p^{2}\left({ }^{3} P\right) 3 d^{4} F$ & 7 & 9.45141 & & 9.37789 & 9.42971 & $9.64-01$ & $1.04+00$ \\
\hline 46 & $2 s^{2} 2 p^{2}\left({ }^{3} P\right) 3 d^{4} F$ & 9 & 9.46046 & & 9.38810 & 9.43880 & $1.19+00$ & $1.26+00$ \\
\hline 47 & $2 s^{2} 2 p^{2}\left({ }^{3} P\right) 3 d^{2} P$ & 3 & 9.47111 & 9.47772 & 9.43876 & 9.47554 & $1.05-02$ & $1.10-02$ \\
\hline 48 & $2 s^{2} 2 p^{2}\left({ }^{3} P\right) 3 d^{2} P$ & 1 & 9.48316 & 9.48337 & 9.45059 & 9.49631 & $1.33-02$ & $1.33-02$ \\
\hline 49 & $2 s^{2} 2 p^{2}\left({ }^{3} P\right) 3 d^{4} D$ & 3 & 9.51013 & 9.52465 & 9.42754 & 9.48952 & $2.45-02$ & $4.40-02$ \\
\hline 50 & $2 s^{2} 2 p^{2}\left({ }^{3} P\right) 3 d^{4} D$ & 1 & 9.51056 & 9.52839 & 9.42836 & 9.48150 & $1.09-01$ & $2.67-02$ \\
\hline 51 & $2 s^{2} 2 p^{2}\left({ }^{3} P\right) 3 d^{4} D$ & 5 & 9.51106 & 9.52465 & 9.43491 & 9.48869 & $4.66-02$ & $1.06-01$ \\
\hline 52 & $2 s^{2} 2 p^{2}\left({ }^{3} P\right) 3 d^{4} D$ & 7 & 9.51542 & & 9.43981 & 9.49314 & $7.01-01$ & $6.50-01$ \\
\hline 53 & $2 s 2 p^{3}\left({ }^{4} S^{\circ}\right) 3 s{ }^{4} S^{\circ}$ & 3 & 9.54080 & 9.53769 & & 9.53436 & $2.07-02$ & $2.05-02$ \\
\hline 54 & $2 \mathrm{~s}^{2} 2 \mathrm{p}^{2}\left({ }^{3} \mathrm{P}\right) 3 \mathrm{~d}^{2} \mathrm{~F}$ & 5 & 9.55293 & 9.53568 & 9.49882 & 9.53486 & $1.05-02$ & $1.19-02$ \\
\hline 55 & $2 s^{2} 2 p^{2}\left({ }^{3} P\right) 3 d^{4} P$ & 5 & 9.55836 & 9.54379 & 9.49706 & 9.54198 & $3.38-03$ & $3.32-03$ \\
\hline 56 & $2 s^{2} 2 p^{2}\left({ }^{3} P\right) 3 d^{4} P$ & 3 & 9.56454 & 9.54999 & 9.50372 & 9.54840 & $3.30-03$ & $3.26-03$ \\
\hline 57 & $2 s^{2} 2 p^{2}\left({ }^{3} P\right) 3 d^{4} P$ & 1 & 9.56781 & 9.55354 & 9.50725 & 9.55174 & $3.27-03$ & $3.22-03$ \\
\hline 58 & $2 s^{2} 2 p^{2}\left({ }^{3} P\right) 3 d^{2} F$ & 7 & 9.57056 & 9.55354 & 9.51731 & 9.55263 & $1.06-02$ & $1.18-02$ \\
\hline
\end{tabular}

Notes. Present results are compared with observed values from NIST compilation (http://physics.nist.gov) and the CI calculations of Landi \& Bhatia (2007) (LB) and Froese Fischer \& Tachiev (2004) (FT). The lifetimes in dipole approximation are also given from the present and Froese Fischer \& Tachiev (2004) (FT) calculations. 
Table 1. continued.

\begin{tabular}{llllccccc}
\hline \hline \multirow{2}{*}{ Index } & State & 2J & \multicolumn{3}{c}{ Excitation energy } & \multicolumn{2}{c}{$\tau(\mathrm{ns})$} \\
& & & Present & Experiment & LB & FT & Present & FT \\
\hline 59 & $2 \mathrm{~s}^{2} 2 \mathrm{p}^{2}\left({ }^{1} \mathrm{~S}\right) 3 \mathrm{p}^{2} \mathrm{P}^{\circ}$ & 3 & 9.63161 & 9.61180 & 9.51835 & & $4.64-01$ & \\
60 & $2 \mathrm{~s}^{2} 2 \mathrm{p}^{2}\left({ }^{1} \mathrm{~S}\right) 3 \mathrm{p}^{2} \mathrm{P}^{\circ}$ & 1 & 9.63264 & 9.61193 & 9.52017 & & $4.61-01$ & \\
61 & $2 \mathrm{~s}^{2} 2 \mathrm{p}^{2}\left({ }^{3} \mathrm{P}\right) 3 \mathrm{~d}^{2} \mathrm{D}$ & 3 & 9.70500 & 9.67811 & 9.64979 & 9.67799 & $5.07-03$ & $5.24-03$ \\
62 & $2 \mathrm{~s}^{2} 2 \mathrm{p}^{2}\left({ }^{3} \mathrm{P}\right) 3 \mathrm{~d}^{2} \mathrm{D}$ & 5 & 9.70995 & 9.68331 & 9.68331 & 9.68303 & $4.88-03$ & $5.02-03$ \\
63 & $2 \mathrm{~s}^{2} 2 \mathrm{p}^{2}\left({ }^{1} \mathrm{D}\right) 3 \mathrm{~d}^{2} \mathrm{G}$ & 7 & 9.89453 & & 9.82671 & 9.85833 & $8.04-03$ & $4.96-02$ \\
64 & $2 \mathrm{~s}^{2} 2 \mathrm{p}^{2}\left({ }^{1} \mathrm{D}\right) 3 \mathrm{~d}^{2} \mathrm{G}$ & 9 & 9.89853 & & 9.82822 & 8.95973 & $9.75-01$ & $1.15+00$ \\
65 & $2 \mathrm{~s}^{2} 2 \mathrm{p}^{2}\left({ }^{1} \mathrm{D}\right) 3 \mathrm{~d}^{2} \mathrm{~F}$ & 7 & 9.90293 & 9.87212 & 9.85442 & 9.87381 & $5.31-03$ & $3.61-03$ \\
66 & $2 \mathrm{~s}^{2} 2 \mathrm{p}^{2}\left({ }^{1} \mathrm{D}\right) 3 \mathrm{~d}^{2} \mathrm{~F}$ & 5 & 9.90341 & 9.87485 & 9.85686 & 9.87648 & $3.21-03$ & $3.36-03$ \\
67 & $2 \mathrm{~s}^{2} 2 \mathrm{p}^{2}\left({ }^{1} \mathrm{D}\right) 3 \mathrm{~d}^{2} \mathrm{D}$ & 3 & 9.93073 & 9.90155 & 9.87498 & 9.90348 & $4.13-03$ & $4.09-03$ \\
68 & $2 \mathrm{~s}^{2} 2 \mathrm{p}^{2}\left({ }^{1} \mathrm{D}\right) 3 \mathrm{~d}^{2} \mathrm{D}$ & 5 & 9.93348 & 9.90474 & 9.87890 & 9.90648 & $4.10-03$ & $4.08-03$ \\
69 & $2 \mathrm{~s}^{2} 2 \mathrm{p}^{2}\left({ }^{1} \mathrm{D}\right) 3 \mathrm{~d}^{2} \mathrm{P}$ & 1 & 10.01161 & 9.96707 & 9.93452 & 9.96865 & $5.19-03$ & $5.31-03$ \\
70 & $2 \mathrm{~s}^{2} 2 \mathrm{p}^{2}\left({ }^{1} \mathrm{D}\right) 3 \mathrm{~d}^{2} \mathrm{P}$ & 3 & 10.01508 & 9.97154 & 9.93894 & 9.97301 & $5.10-03$ & $5.22-03$ \\
71 & $2 \mathrm{~s} 2 \mathrm{p}^{3}\left({ }^{4} \mathrm{~S}^{\circ}\right) 3 \mathrm{p}^{4} \mathrm{P}$ & 5 & 10.02183 & & & 10.02158 & $2.11-02$ & $2.21-02$ \\
72 & $2 \mathrm{~s} 2 \mathrm{p}^{3}\left({ }^{4} \mathrm{~S}^{\circ}\right) 3 \mathrm{p}{ }^{4} \mathrm{P}$ & 3 & 10.02297 & & & 10.022711 & $2.14-02$ & $2.24-02$ \\
73 & $2 \mathrm{~s} 2 \mathrm{p}^{3}\left({ }^{4} \mathrm{~S} \mathrm{~S}^{\circ}\right) 3 \mathrm{p}{ }^{4} \mathrm{P}$ & 1 & 10.02366 & & & 10.02348 & $2.15-02$ & $2.26-02$ \\
74 & $2 \mathrm{~s}^{2} 2 \mathrm{p}^{2}\left({ }^{1} \mathrm{D}\right) 3 \mathrm{~d}^{2} \mathrm{~S}$ & 1 & 10.03347 & 10.01650 & 9.99674 & 10.01845 & $6.82-03$ & $6.73-03$ \\
\hline
\end{tabular}



Fig. 1. Ratio between the present velocity and length oscillator strengths is shown as a function of length oscillator strengths. The dotted lines indicate $20 \%$ deviation.

dipole-allowed transitions. The agreement between the length and velocity forms of oscillator strengths may to some extent indicate the accuracy of wave functions and convergence of CI expansions. However, it is not a sufficient condition for the accuracy of results. The convergence of results is an important accuracy criterion. We have plotted ratio of the present velocity and length values as a function of length oscillator strengths in Fig. 1. It is clear from Fig. 1 that there is normally a very good agreement between the present length and velocity forms of oscillator strengths, particularly for transitions with significant strengths. The weak transitions, however, are not so accurate. The weak transitions belong mainly to the intercombination transitions. The intercombination transitions occur due to spinorbit mixing of total terms. This mixing strongly depends on the details of calculation and it is difficult to obtain the convergent results in some cases.

We begin our discussion of collision results for the excitation collision strengths for the forbidden $2 \mathrm{~s}^{2} 2 \mathrm{p}^{3}{ }^{4} \mathrm{~S}_{3 / 2}^{\circ}-{ }^{2} \mathrm{D}_{5 / 2}^{\circ}$ (1-2) transition in both closed-channel and all open-channel energy regions in Fig. 2. The collision strengths show extensive
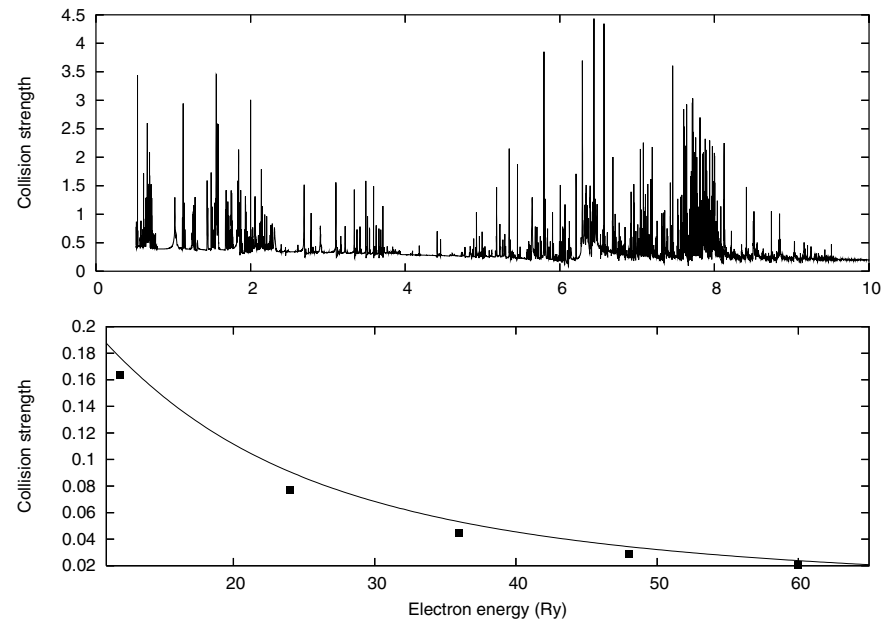

Fig. 2. Collision strengths for the forbidden $2 s^{2} 2 p^{3}{ }^{4} S_{3 / 2}^{\circ}-{ }^{2} D_{5 / 2}^{\circ}(1-2)$ transition as a function of incident electron energy. Upper panel, resonant collision strengths in the closed channel region; lower panel, nonresonant collision strengths in all open channels region and present results (solid curve) are compared with the calculation of Landi \& Bhatia (2007) (solid rectangles).

resonance structures which are typical for low-energy electron scattering on ions in closed-channel region. These resonances are expected to contribute significantly to the collision rates at low temperatures. Note also that our collision strengths show a richer resonance structure with many narrow resonances. It is partly because our results directly include the fine-structure splitting both for the $N$-electron target states and for $(N+1)$-electron resonances. Our calculation predicts very smooth behavior at the above-threshold region for the forbidden $2 \mathrm{~s}^{2} 2 \mathrm{p}^{3}{ }^{4} \mathrm{~S}_{3 / 2}^{\circ}{ }^{2} \mathrm{D}_{5 / 2}^{\circ}$ (1-2) transition plotted in the lower panel of Fig. 2. Our results for non-resonant collision strengths have been compared with the available distorted-wave calculation of Landi \& Bhatia (2007) who reported results at five incident electron energies. The present results are shown by solid curve, while the solid rectangles display the results of Landi \& Bhatia (2007). There is a good agreement between the two calculations with a maximum deviation of about $10 \%$. 
Table 2. Comparison of oscillator strengths and transition probabilities $\left(\mathrm{s}^{-1}\right)$ for some dipole radiative E1 transitions with the calculations of Landi \& Bhatia (2007) (LB) and Froese Fischer \& Tachiev (2004) (FT).

\begin{tabular}{|c|c|c|c|c|c|c|c|c|}
\hline \multirow[t]{2}{*}{ Initial level } & \multirow[t]{2}{*}{$g_{i}$} & \multirow[t]{2}{*}{$g_{f}$} & \multicolumn{2}{|c|}{ Present } & \multicolumn{2}{|c|}{ LB } & \multicolumn{2}{|c|}{ FT } \\
\hline & & & $f_{i f}$ & $A_{f i}$ & $f_{i f}$ & $A_{f i}$ & $f_{i f}$ & $A_{f i}$ \\
\hline $2 s^{2} 2 p^{3}{ }^{4} S^{\circ}-2 s 2 p^{4}{ }^{4} P$ & 4 & 6 & $1.026-01$ & $2.847+09$ & $1.146-01$ & $3.137+09$ & $1.013-01$ & $2.783+09$ \\
\hline $2 s^{2} 2 p^{3}{ }^{4} s^{\circ}-2 s 2 p^{4}{ }^{4} P$ & 4 & . & $896-02$ & $2.905+09$ & $7.700-02$ & $3.205+09$ & $6.805-02$ & $2.842+09$ \\
\hline $2 s^{2} 2 p^{3}{ }^{4} S^{\circ}-2 s 2 p^{4}{ }^{4} P$ & 4 & 2 & $.464-02$ & $2.940+09$ & $3.865-02$ & $3.244+09$ & $3.418-02$ & $2.874+09$ \\
\hline $2 s^{2} 2 p^{3}{ }^{4} S^{\circ}-2 s 2 p^{4}{ }^{2} D$ & 4 & 6 & $9.936-08$ & $5.266+03$ & $9.992-08$ & $5.576+03$ & $1.640-07$ & $8.580+03$ \\
\hline $2 s^{2} 2 p^{3}{ }^{4} s^{\circ}-2 s 2 p^{4}{ }^{2} D$ & 4 & 4 & $.308-08$ & $4.220+03$ & $1.496-07$ & $1.253+04$ & $1.009-07$ & $7.924+03$ \\
\hline $2 s^{2} 2 p^{3}{ }^{4} S^{\circ}-2 s 2 p^{4}{ }^{2} S$ & 4 & 2 & $.020-05$ & $2.265+06$ & $1.539-05$ & $3.525+06$ & $1.033-05$ & $2.239+06$ \\
\hline $2 s^{2} 2 p^{3}{ }^{4} S^{\circ}-2 s 2 p^{4}{ }^{2} P$ & 4 & 4 & $3.461-05$ & $4.266+06$ & $3.915-05$ & $5.207+06$ & $3.683-05$ & $4.486+06$ \\
\hline $2 s^{2} 2 p^{3}{ }^{4} S^{\circ}-2 s 2 p^{4}{ }^{2} P$ & 4 & 2 & $6.293-06$ & $1.565+06$ & $5.752-06$ & $1.545+06$ & $5.499-06$ & $1.352+06$ \\
\hline $2 s^{2} 2 p^{3}{ }^{4} S^{\circ}-2 s^{2} 2 p^{2}\left({ }^{3} P\right) 3 s^{4} P$ & 4 & 2 & $004-02$ & $2.136+10$ & $2.069-02$ & $2.218+10$ & $2.071-02$ & $2.205+10$ \\
\hline $2 s^{2} 2 p^{3}{ }^{4} S^{\circ}-2 s^{2} 2 p^{2}\left({ }^{3} P\right) 3 s^{4} P$ & 4 & 4 & $04-02$ & $2.138+10$ & $4.125-02$ & $2.216+10$ & $4.155-02$ & $2.217+10$ \\
\hline $2 s^{2} 2 p^{3}{ }^{4} s^{\circ}-2 s^{2} 2 p^{2}\left({ }^{3} P\right) 3 s^{4} P$ & 4 & 0 & $01-02$ & $2.144+10$ & $6.157-02$ & $2.212+10$ & $267-02$ & $2.237+10$ \\
\hline $2 s^{2} 2 p^{3}{ }^{4} S^{\circ}-2 s^{2} 2 p^{2}\left({ }^{3} P\right) 3 s^{2} P$ & 4 & 2 & 66 & $6.124+06$ & $5.677-06$ & $6.297+06$ & & $8.627+06$ \\
\hline $2 s^{2} 2 p^{3}{ }^{4} S^{\circ}-2 s^{2} 2 p^{2}\left({ }^{3} P\right) 3 s^{2} P$ & 4 & 4 & & & & & & $68+07$ \\
\hline $2 s^{2} 2 p^{3}{ }^{4} S^{\circ}-2 s^{2} 2 p^{2}\left({ }^{1} D\right) 3 s^{2} D$ & 4 & 0 & $28-05$ & & & & & $573+06$ \\
\hline $2 s^{2} 2 p^{3}{ }^{4} S^{\circ}-2 s^{2} 2 p^{2}\left({ }^{1} D\right) 3 s^{2} D$ & 4 & 4 & 06 & & & & & $1.657+06$ \\
\hline $2 s^{2} 2 p^{3}{ }^{4} S^{\circ}-2 s^{2} 2 p^{2}\left({ }^{1} S\right) 3 s^{2} S$ & 4 & 2 & 8 & & 773 & & & $35+04$ \\
\hline $2 s^{2} 2 p^{3}{ }^{4} S^{\circ}-2 s^{2} 2 p^{2}\left({ }^{3} P\right) 3 d^{4} F$ & 4 & 4 & 5 & & & & & \\
\hline $2 s^{2} 2 p^{3}{ }^{4} S^{\circ}-2 s^{2} 2 p^{2}\left({ }^{3} P\right) 3 d^{4} F$ & 4 & 6 & & & & & & -07 \\
\hline $2 s^{2} 2 p^{3}{ }^{4} S^{\circ}-2 s^{2} 2 p^{2}\left({ }^{3} P\right) 3 d^{2} P$ & 4 & 4 & & & & & & $31+09$ \\
\hline $2 s^{2} 2 p^{3}{ }^{4} S^{\circ}-2 s^{2} 2 p^{2}\left({ }^{3} P\right) 3 d^{4} D$ & 4 & 2 & & & & & & $157+09$ \\
\hline $2 s^{2} 2 p^{3}{ }^{4} S^{\circ}-2 s^{2} 2 p^{2}\left({ }^{3} P\right) 3 d^{4} D$ & 4 & 6 & 02 & & & & & $126+09$ \\
\hline $2 s^{2} 2 p^{3}{ }^{4} S^{\circ}-2 s^{2} 2 p^{2}\left({ }^{3} P\right) 3 d^{4} D$ & 4 & 4 & & & & & & $307+09$ \\
\hline $2 s^{2} 2 p^{3}{ }^{4} S^{\circ}-2 s^{2} 2 p^{2}\left({ }^{3} P\right) 3 d^{2} P$ & 4 & 2 & & & & & & $087+08$ \\
\hline $2 s^{2} 2 p^{3}{ }^{4} S^{\circ}-2 s^{2} 2 p^{2}\left({ }^{3} P\right) 3 d^{4} P$ & 4 & 6 & & & & & & +11 \\
\hline $2 s^{2} 2 p^{3}{ }^{4} S^{\circ}-2 s^{2} 2 p^{2}\left({ }^{3} P\right) 3 d^{2} F$ & 4 & 6 & & & & & & \\
\hline $2 s^{2} 2 p^{3}{ }^{4} S^{\circ}-2 s^{2} 2 p^{2}\left({ }^{3} P\right) 3 d^{4} P$ & 4 & 4 & & & & & & $3.054+11$ \\
\hline $2 s^{2} 2 p^{3}{ }^{4} S^{\circ}-2 s^{2} 2 p^{2}\left({ }^{3} P\right) 3 d^{4} P$ & 4 & 2 & & 11 & & 11 & & $95+11$ \\
\hline $2 s^{2} 2 p^{3}{ }^{4} S^{\circ}-2 s^{2} 2 p^{2}\left({ }^{3} P\right) 3 d^{2} D$ & 4 & 4 & & & & & & +05 \\
\hline $2 s^{2} 2 p^{3}{ }^{4} s^{\circ}-2 s^{2} 2 p^{2}\left({ }^{3} P\right) 3 d^{2} D$ & 4 & 6 & & & & & & +06 \\
\hline $2 \mathrm{~s}^{2} 2 \mathrm{p}^{3}{ }^{4} \mathrm{~S}^{\circ}-2 \mathrm{~s}^{2} 2 \mathrm{p}^{2}\left({ }^{1} \mathrm{D}\right)$ & 4 & 6 & & & & & & $6+05$ \\
\hline $2 s^{2} 2 p^{3}{ }^{4} S^{\circ}-2 s^{2} 2 p^{2}\left({ }^{1} D\right) 3 d^{2} D$ & 4 & 4 & & & & & & $1.126+07$ \\
\hline $2 s^{2} 2 p^{3}{ }^{4} S^{\circ}-2 s^{2} 2 p^{2}\left({ }^{1} D\right) 3$ & 4 & 6 & & & & & 04 & $28+07$ \\
\hline $2 s^{2} 2 p^{3}{ }^{4} s^{\circ}-2 s^{2} 2 p^{2}\left({ }^{1} D\right)$ & 4 & 2 & 5 & & 06 & 07 & 05 & +07 \\
\hline $2 \mathrm{~s}^{2} 2 \mathrm{p}^{3}{ }^{4} \mathrm{~s}^{\circ}-2 \mathrm{~s}^{2} 2 \mathrm{p}^{2}\left({ }^{1} \mathrm{D}\right)$ & 4 & 4 & & & -05 & 07 & 04 & +07 \\
\hline $2 s^{2} 2 p^{3}{ }^{4} S^{\circ}-2 s^{2} 2 p^{2}\left({ }^{1} D\right) 3$ & 4 & 2 & & 4.2 & $1.501-05$ & $2.443+07$ & 05 & +08 \\
\hline $2 s^{2} 2 p^{3}{ }^{4} S^{\circ}-2 s 2 p^{3}\left({ }^{4} S^{\circ}\right) 31$ & 4 & 6 & 02 & 4.6 & & & 02 & -10 \\
\hline $2 s^{2} 2 p^{3}{ }^{4} S^{\circ}-2 s 2 p^{3}\left({ }^{4} S^{\circ}\right)$ & 4 & 4 & & 4. & & & & -10 \\
\hline $2 s^{2} 2 p^{3}{ }^{4} S^{\circ}-2 s 2 p^{3}\left({ }^{4} S^{\circ}\right)$ & 4 & 2 & 2 & $5+10$ & & & 02 & +10 \\
\hline $2 s^{2} 2 p^{32} D^{\circ}-2 s 2 p^{4}{ }^{4} P$ & 6 & 6 & & 05 & 05 & +05 & & $2+05$ \\
\hline $2 \mathrm{~s}^{2} 2 \mathrm{p}^{3}{ }^{2} \mathrm{D}^{\circ}-2 \mathrm{~s} 2 \mathrm{p}^{4}{ }^{4} \mathrm{P}$ & 6 & 4 & & 04 & -07 & 04 & 07 & $76+04$ \\
\hline $2 \mathrm{~s}^{2} 2 \mathrm{p}^{3}{ }^{2} \mathrm{D}^{\circ}-2 \mathrm{~s} 2 \mathrm{p}^{4}{ }^{2} \mathrm{D}$ & 6 & 6 & & & -01 & $4+09$ & 01 & $6.139+09$ \\
\hline $2 \mathrm{~s}^{2} 2 \mathrm{p}^{3}{ }^{2} \mathrm{D}^{\circ}-2 \mathrm{~s} 2 \mathrm{p}^{4}{ }^{2} \mathrm{D}$ & 6 & 4 & & & -03 & +08 & & $12+08$ \\
\hline $2 \mathrm{~s}^{2} 2 \mathrm{p}^{3}{ }^{2} \mathrm{D}^{\circ}-2 \mathrm{~s} 2 \mathrm{p}^{4}{ }^{2} \mathrm{P}$ & 6 & 4 & & & & & & $7+10$ \\
\hline $2 s^{2} 2 p^{3}{ }^{2} D^{\circ}-2 s^{2} 2 p^{2}\left({ }^{3} P\right) 3 s^{4} P$ & 6 & 4 & & & & 07 & & $72+07$ \\
\hline $2 s^{2} 2 p^{3}{ }^{2} D^{\circ}-2 s^{2} 2 p^{2}\left({ }^{3} P\right) 3 s^{4} P$ & 6 & 6 & $86-05$ & 2.8 & $6.978-05$ & $9+07$ & & $08+07$ \\
\hline $2 s^{2} 2 p^{32} D^{\circ}-2 s^{2} 2 p^{2}\left({ }^{3} P\right) 3 s^{2} P$ & 6 & 4 & $99-02$ & 3. & $3-02$ & $4+10$ & & $14+10$ \\
\hline $2 s^{2} 2 p^{3}{ }^{2} D^{\circ}-2 s^{2} 2 p^{2}\left({ }^{1} D\right) 3 s^{2} D$ & 6 & 6 & $5.218-02$ & $2.716+10$ & -02 & 10 & & $04+10$ \\
\hline $2 s^{2} 2 p^{3}{ }^{2} D^{\circ}-2 s^{2} 2 p^{2}\left({ }^{1} D\right) 3 s^{2} D$ & 6 & 4 & $2.695-03$ & 2.105 & & 09 & & $4+09$ \\
\hline $2 s^{2} 2 p^{3}{ }^{2} D^{\circ}-2 s^{2} 2 p^{2}\left({ }^{3} P\right) 3 d^{4} F$ & 6 & 4 & $9.818-07$ & $9.416+05$ & $53-07$ & $3+05$ & & $84+05$ \\
\hline $2 s^{2} 2 p^{3}{ }^{2} D^{\circ}-2 s^{2} 2 p^{2}\left({ }^{3} P\right) 3 d^{4} F$ & 6 & 6 & $1.217-04$ & $7.787+07$ & $1.099-04$ & 07 & & $0+07$ \\
\hline $2 s^{2} 2 p^{3}{ }^{2} D^{\circ}-2 s^{2} 2 p^{2}\left({ }^{3} P\right) 3 d^{4} F$ & 6 & 8 & $3.933-04$ & $1.891+08$ & $3.633-04$ & 08 & & $33+08$ \\
\hline $2 s^{2} 2 p^{3}{ }^{2} D^{\circ}-2 s^{2} 2 p^{2}\left({ }^{3} P\right) 3 d^{2} P$ & 6 & 4 & $1.635-02$ & $1.587+10$ & $1.447-02$ & 10 & & $82+10$ \\
\hline $2 s^{2} 2 p^{3}{ }^{2} D^{\circ}-2 s^{2} 2 p^{2}\left({ }^{3} P\right) 3 d^{4} D$ & 6 & 6 & $3.245-05$ & $2.106+07$ & $4.710-05$ & $3.038+07$ & $1.672-05$ & $083+07$ \\
\hline $2 s^{2} 2 p^{3}{ }^{2} D^{\circ}-2 s^{2} 2 p^{2}\left({ }^{3} P\right) 3 d^{4} D$ & 6 & 4 & $2.571-03$ & $2.504+09$ & $6.400-03$ & $6.186+09$ & $3.159-03$ & $3.070+09$ \\
\hline $2 s^{2} 2 p^{3}{ }^{2} D^{\circ}-2 s^{2} 2 p^{2}\left({ }^{3} P\right) 3 d^{4} D$ & 6 & 8 & $9.452-04$ & $4.606+08$ & 6.611-04 & $3.202+08$ & $1.295-03$ & $6.300+08$ \\
\hline $2 s^{2} 2 p^{3}{ }^{2} D^{\circ}-2 s^{2} 2 p^{2}\left({ }^{3} P\right) 3 d^{4} P$ & 6 & 6 & $5.708-05$ & $3.749+07$ & $1.108-04$ & $7.256+07$ & $6.329-05$ & $4.148+07$ \\
\hline $2 s^{2} 2 p^{3}{ }^{2} D^{\circ}-2 s^{2} 2 p^{2}\left({ }^{3} P\right) 3 d^{2} F$ & 6 & 6 & $9.390-03$ & $6.170+09$ & $9.073-03$ & $5.936+09$ & $8.516-03$ & $5.574+09$ \\
\hline $2 s^{2} 2 p^{3}{ }^{2} D^{\circ}-2 s^{2} 2 p^{2}\left({ }^{3} P\right) 3 d^{4} P$ & 6 & 4 & $8.233-05$ & $8.123+07$ & $8.638-05$ & $8.495+07$ & $8.574-05$ & $8.442+07$ \\
\hline $2 s^{2} 2 p^{3}{ }^{2} D^{\circ}-2 s^{2} 2 p^{2}\left({ }^{3} P\right) 3 d^{2} F$ & 6 & 8 & $1.890-01$ & $9.351+10$ & $1.783-01$ & $8.785+10$ & $1.710-01$ & $8.426+10$ \\
\hline $2 s^{2} 2 p^{3}{ }^{2} D^{\circ}-2 s^{2} 2 p^{2}\left({ }^{3} P\right) 3 d^{2} D$ & 6 & 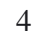 & $9.300-03$ & $9.449+09$ & $1.058-02$ & $1.076+10$ & $9.535-03$ & $9.660+09$ \\
\hline $2 s^{2} 2 p^{3}{ }^{2} D^{\circ}-2 s^{2} 2 p^{2}\left({ }^{3} P\right) 3 d^{2} D$ & 6 & 6 & $1.106-01$ & $7.500+10$ & $1.274-01$ & $8.651+10$ & $1.117-01$ & $7.553+10$ \\
\hline
\end{tabular}


Table 2. continued.

\begin{tabular}{lccccccccc}
\hline \hline Initial level Final level & \multirow{2}{*}{$g_{i}$} & \multicolumn{2}{c}{$g_{f}$} & \multicolumn{2}{c}{ Present } & \multicolumn{2}{c}{ LB } & \multicolumn{2}{c}{ FT } \\
& & & $f_{i f}$ & $A_{f i}$ & $f_{i f}$ & $A_{f i}$ & $f_{i f}$ & $A_{f i}$ \\
\hline $2 s^{2} 2 p^{3}{ }^{2} \mathrm{D}^{\circ}-2 \mathrm{~s}^{2} 2 \mathrm{p}^{2}\left({ }^{1} \mathrm{D}\right) 3 \mathrm{~d}^{2} \mathrm{~F}$ & 6 & 8 & $3.534-01$ & $1.875+11$ & $6.980-01$ & $3.703+11$ & $5.226-01$ & $2.761+11$ \\
$2 s^{2} 2 p^{3}{ }^{2} \mathrm{D}^{\circ}-2 \mathrm{~s}^{2} 2 \mathrm{p}^{2}\left({ }^{1} \mathrm{D}\right) 3 \mathrm{~d}^{2} \mathrm{~F}$ & 6 & 6 & $6.465-02$ & $4.581+10$ & $7.808-02$ & $5.528+10$ & $5.370-02$ & $3.785+10$ \\
$2 s^{2} 2 p^{3}{ }^{2} \mathrm{D}^{\circ}-2 \mathrm{~s}^{2} 2 \mathrm{p}^{2}\left({ }^{1} \mathrm{D}\right) 3 \mathrm{~d}^{2} \mathrm{G}$ & 6 & 8 & $2.325-01$ & $1.236+11$ & $4.673-03$ & $2.455+09$ & $3.666-02$ & $1.930+10$ \\
$2 s^{2} 2 p^{3}{ }^{2} \mathrm{D}^{\circ}-2 \mathrm{~s}^{2} 2 \mathrm{p}^{2}\left({ }^{1} \mathrm{D}\right) 3 \mathrm{~d}^{2} \mathrm{D}$ & 6 & 4 & $1.527-02$ & $1.629+10$ & $1.579-02$ & $1.683+10$ & $1.574-02$ & $1.674+10$ \\
$2 s^{2} 2 p^{3}{ }^{2} \mathrm{D}^{\circ}-2 \mathrm{~s}^{2} 2 \mathrm{p}^{2}\left({ }^{1} \mathrm{D}\right) 3 \mathrm{~d}^{2} \mathrm{D}$ & 6 & 6 & $1.514-01$ & $1.077+11$ & $1.514-01$ & $1.077+11$ & $1.659-01$ & $1.177+11$ \\
$2 s^{2} 2 p^{3}{ }^{2} \mathrm{D}^{\circ}-2 \mathrm{~s}^{2} 2 \mathrm{p}^{2}\left({ }^{1} \mathrm{D}\right) 3 \mathrm{~d}^{2} \mathrm{P}$ & 6 & 4 & $4.506-02$ & $4.878+10$ & $4.405-02$ & $4.756+10$ & $4.639-02$ & $5.007+10$ \\
$2 s^{2} 2 p^{3}{ }^{2} \mathrm{D}^{\circ}-2 \mathrm{~s} 2 \mathrm{p}^{3}\left({ }^{4} \mathrm{~S}^{\circ}\right) 3 \mathrm{p}^{4} \mathrm{P}$ & 6 & 6 & $1.152-06$ & $8.429+05$ & & & $1.539-06$ & $1.118+06$ \\
$2 s^{2} 2 p^{3}{ }^{2} \mathrm{D}^{\circ}-2 \mathrm{~s} 2 \mathrm{p}^{3}\left({ }^{4} \mathrm{~S}^{\circ}\right) 3 \mathrm{p} \mathrm{p}^{4} \mathrm{P}$ & 6 & 4 & $1.932-06$ & $2.121+06$ & & & $4.211-06$ & $4.593+06$ \\
\hline
\end{tabular}

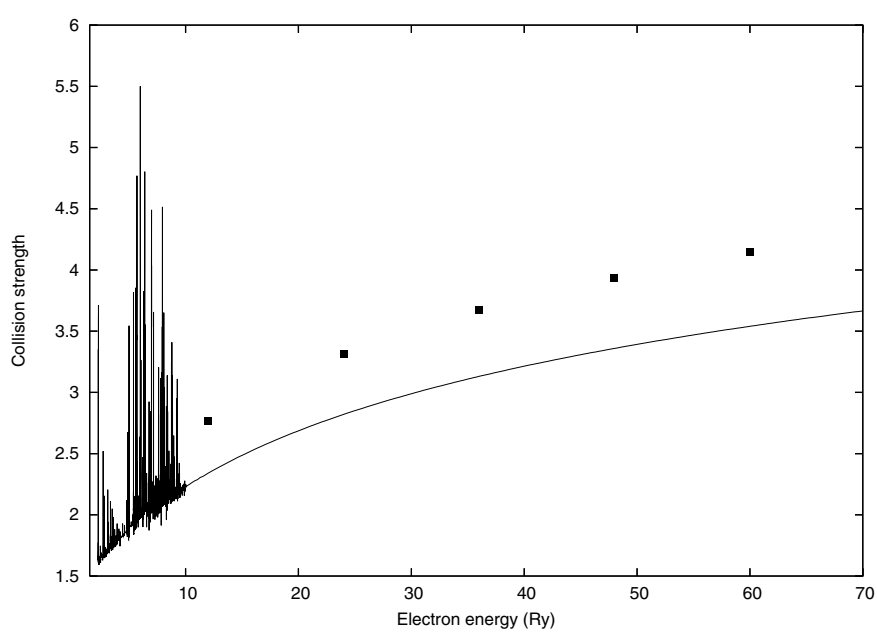

Fig. 3. Collision strengths for the dipole-allowed $2 s^{2} 2 p^{3}{ }^{4} S_{3 / 2}^{\circ}$ $2 \mathrm{~s} 2 \mathrm{p}^{4}{ }^{4} \mathrm{P}_{5 / 2}(1-6)$ transition as a function of incident electron energy from threshold to $70 \mathrm{Ry}$. Solid curve, present calculation; solid rectangles, calculation of Landi \& Bhatia (2007).

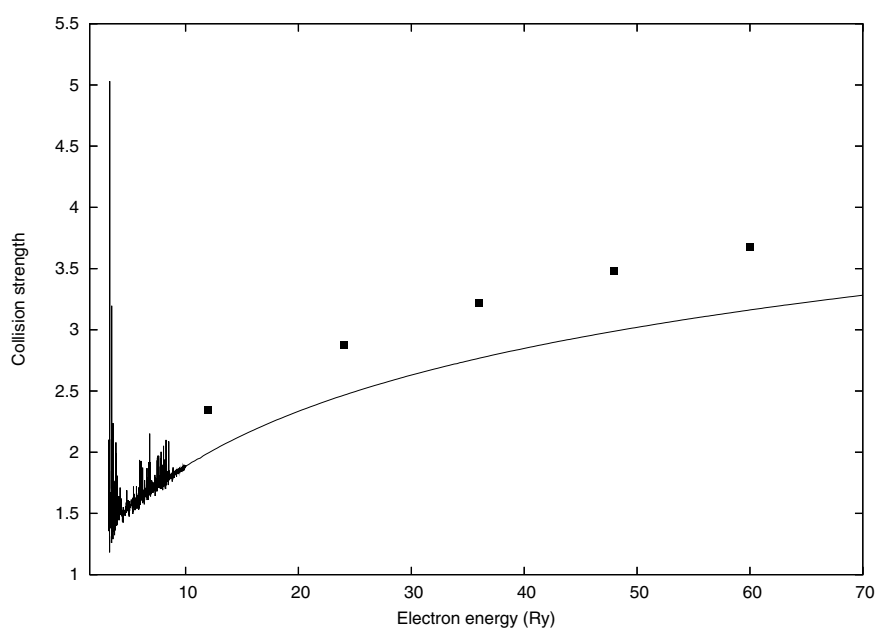

Fig. 4. Collision strengths for the dipole-allowed $2 \mathrm{~s}^{2} 2 \mathrm{p}^{3}{ }^{2} \mathrm{D}_{3 / 2}^{\circ}-2 \mathrm{~s} 2 \mathrm{p}^{4}$ ${ }^{2} \mathrm{D}_{3 / 2}$ (3-10) transition as a function of incident electron energy from threshold to 70 Ry. Solid curve, present calculation; solid rectangles, calculation of Landi \& Bhatia (2007).

The collision strengths for the dipole-allowed $2 \mathrm{~s}^{2} 2 \mathrm{p}^{3}$ ${ }^{4} \mathrm{~S}_{3 / 2}^{\circ}-2 \mathrm{~s} 2 \mathrm{p}^{4}{ }^{4} \mathrm{P}_{5 / 2} \quad(1-6), 2 \mathrm{~s}^{2} 2 \mathrm{p}^{3}{ }^{2} \mathrm{D}_{3 / 2}^{\circ}-2 \mathrm{~s} 2 \mathrm{p}^{4} \quad{ }^{2} \mathrm{D}_{3 / 2} \quad(3-10)$, $2 \mathrm{~s}^{2} 2 \mathrm{p}^{3} \quad{ }^{2} \mathrm{P}_{1 / 2}^{\circ}-2 \mathrm{~s}^{2} 2 \mathrm{p}^{2}\left({ }^{1} \mathrm{D}\right) 3 \mathrm{~s} \quad{ }^{2} \mathrm{D}_{3 / 2} \quad(4-22), \quad 2 \mathrm{~s}^{2} 2 \mathrm{p}^{3} \quad{ }^{4} \mathrm{~S}_{3 / 2}^{\circ}$ $2 \mathrm{~s}^{2} 2 \mathrm{p}^{2}\left({ }^{3} \mathrm{P}\right) 3 \mathrm{~d}{ }^{4} \mathrm{P}_{5 / 2}$ (1-55), and $2 \mathrm{~s}^{2} 2 \mathrm{p}^{3}{ }^{2} \mathrm{D}_{3 / 2}^{\circ}-2 \mathrm{~s}^{2} 2 \mathrm{p}^{2}\left({ }^{1} \mathrm{D}\right) 3 \mathrm{~d}$ ${ }^{2} \mathrm{~F}_{5 / 2}$ (3-66) transitions are displayed in both resonant and

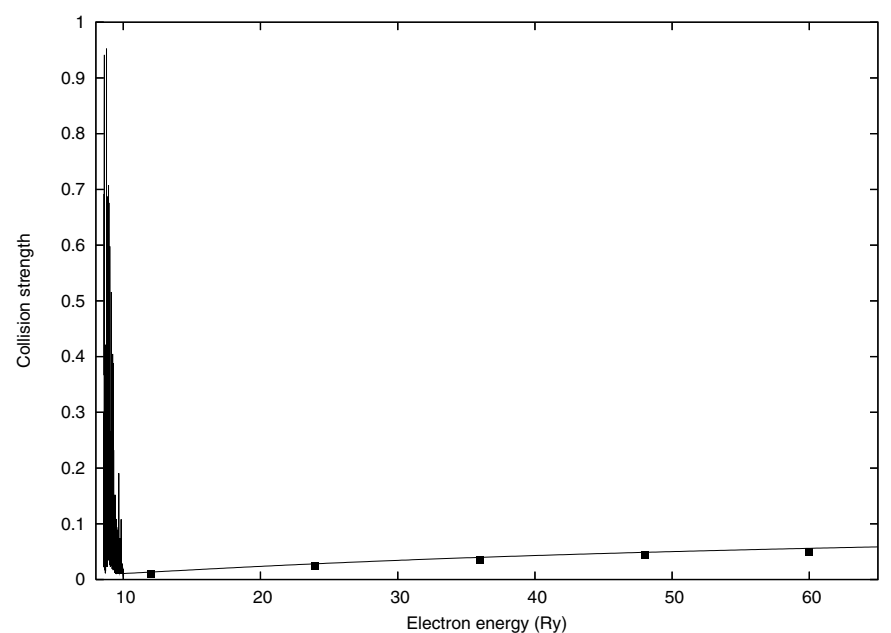

Fig. 5. Collision strengths for the dipole-allowed $2 s^{2} 2 \mathrm{p}^{3} \quad{ }^{2} \mathrm{P}_{1 / 2}^{\circ}$ $2 s^{2} 2 p^{2}\left({ }^{1} \mathrm{D}\right) 3 \mathrm{~s}^{2} \mathrm{D}_{3 / 2}(4-22)$ transition as a function of incident electron energy from threshold to $65 \mathrm{Ry}$. Solid curve, present calculation; solid rectangles, calculation of Landi \& Bhatia (2007).

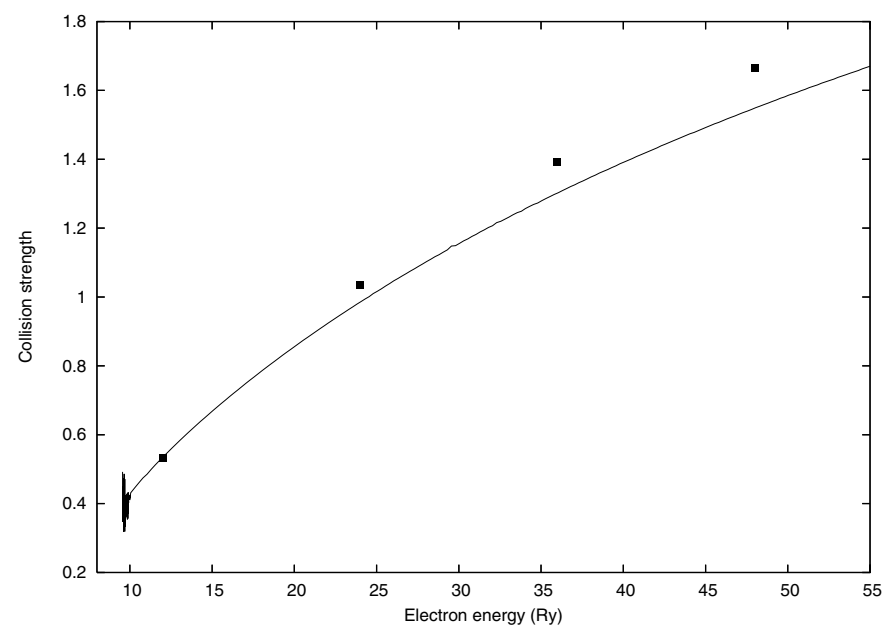

Fig. 6. Collision strengths for the dipole-allowed $2 \mathrm{~s}^{2} 2 \mathrm{p}^{3}{ }^{4} \mathrm{~S}_{3 / 2}^{\circ}$ $2 s^{2} 2 p^{2}\left({ }^{3} \mathrm{P}\right) 3 d^{4} \mathrm{P}_{5 / 2}(1-55)$ transition as a function of incident electron energy from threshold to $55 \mathrm{Ry}$. Solid curve, present calculation; solid rectangles, calculation of Landi \& Bhatia (2007).

non-resonant regions in Figs. 3-7. Comparison of our results with the collision strengths from the distorted-wave calculations by Landi \& Bhatia (2007) shows an overall good agreement for the background collision strength of $2 \mathrm{~s}^{2} 2 \mathrm{p}^{3}{ }^{2} \mathrm{P}_{1 / 2}^{\circ}-2 \mathrm{~s}^{2} 2 \mathrm{p}^{2}\left({ }^{1} \mathrm{D}\right) 3 \mathrm{~s}$ ${ }^{2} \mathrm{D}_{3 / 2}(4-22)$ and $2 \mathrm{~s}^{2} 2 \mathrm{p}^{3}{ }^{4} \mathrm{~S}_{3 / 2}^{\circ}-2 \mathrm{~s}^{2} 2 \mathrm{p}^{2}\left({ }^{3} \mathrm{P}\right) 3 \mathrm{~d}{ }^{4} \mathrm{P}_{5 / 2}$ (1-55) transitions, but some significant differences exist for the other 


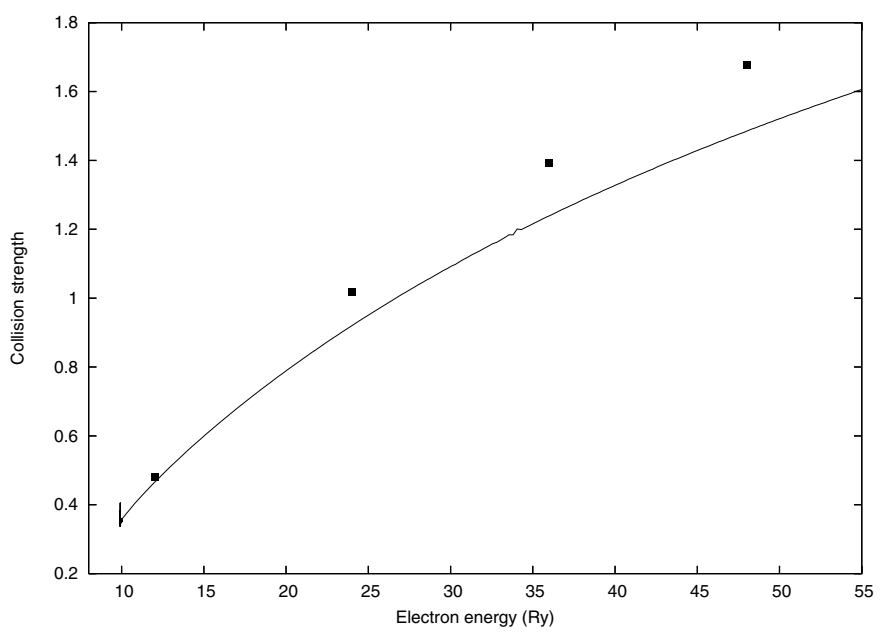

Fig. 7. Collision strengths for the dipole-allowed $2 s^{2} 2 \mathrm{p}^{3}{ }^{2} \mathrm{D}_{3 / 2}^{\circ}$ $2 s^{2} 2 p^{2}\left({ }^{1} \mathrm{D}\right) 3 \mathrm{~d}^{2} \mathrm{~F}_{5 / 2}(3-66)$ transition as a function of incident electron energy from threshold to $55 \mathrm{Ry}$. Solid curve, present calculation; solid rectangles, calculation of Landi \& Bhatia (2007).
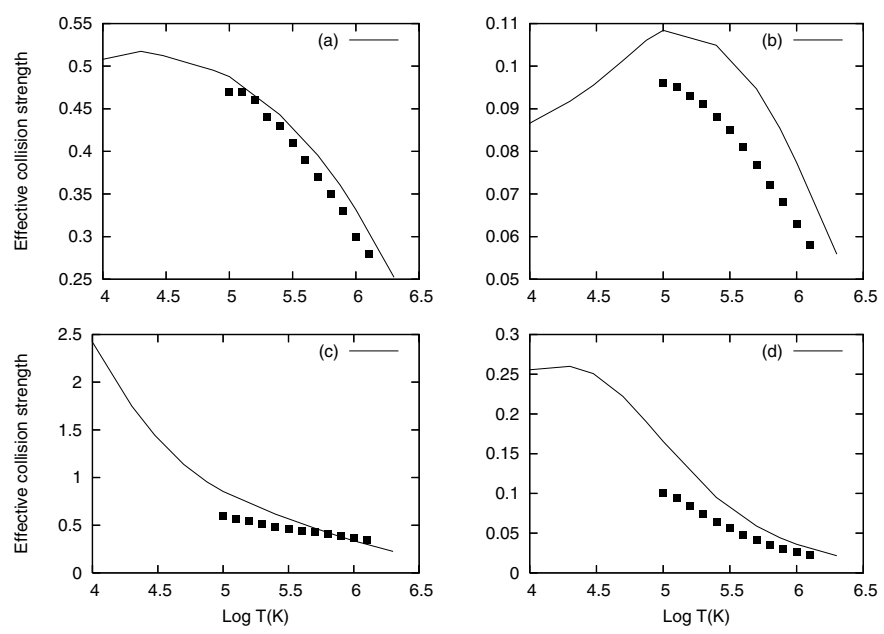

Fig. 8. Effective collision strengths for the forbidden a) $2 s^{2} 2 p^{3}{ }^{4} S_{3 / 2}^{\circ}$ ${ }^{2} \mathrm{D}_{5 / 2}^{\circ}(1-2)$, b) $2 \mathrm{~s}^{2} 2 \mathrm{p}^{3}{ }^{4} \mathrm{~S}_{3 / 2}^{\circ}-{ }^{2} \mathrm{P}_{1 / 2}^{\circ}(1-4)$, c) $2 \mathrm{~s}^{2} 2 \mathrm{p}^{3}{ }^{2} \mathrm{P}_{1 / 2}^{\circ}-{ }^{2} \mathrm{P}_{3 / 2}^{\circ}$ (4-5), and d) $2 \mathrm{~s}^{2} 2 \mathrm{p}^{3}{ }^{4} \mathrm{~S}_{3 / 2}^{\circ}-2 \mathrm{~s}^{2} 2 \mathrm{p}^{2}\left({ }^{3} \mathrm{P}\right) 3 \mathrm{~s}^{2} \mathrm{P}_{3 / 2}(1-20)$ transitions as a function of electron temperature. Present results, solid curve; Ramsbottom \& Bell (1997), solid rectangles.

three dipole-allowed $2 \mathrm{~s}^{2} 2 \mathrm{p}^{3}{ }^{4} \mathrm{~S}_{3 / 2}^{\circ}-2 \mathrm{~s} 2 \mathrm{p}^{4}{ }^{4} \mathrm{P}_{5 / 2}$ (1-6), $2 \mathrm{~s}^{2} 2 \mathrm{p}^{3}$ ${ }^{2} \mathrm{D}_{3 / 2}^{\circ}-2 \mathrm{~s} 2 \mathrm{p}^{4}{ }^{2} \mathrm{D}_{3 / 2} \quad(3-10)$, and $2 \mathrm{~s}^{2} 2 \mathrm{p}^{3} \quad{ }^{2} \mathrm{D}_{3 / 2}^{\circ}-2 \mathrm{~s}^{2} 2 \mathrm{p}^{2}\left({ }^{1} \mathrm{D}\right) 3 \mathrm{~d}$ ${ }^{2} \mathrm{~F}_{5 / 2}$ (3-66) transitions. The collision strengths for the dipoleallowed transitions show expected trend at higher energies. The collision strengths for stronger dipole-allowed transitions increase rapidly at higher energies than for the weaker dipoleallowed transitions. There is an agreement in shape among the two theories but some discrepancies exist in the magnitude of collision strengths. Our results are consistently lower than the calculation of Landi \& Bhatia (2007) for the four out of five transitions shown in Figs. 3-7. Most likely, the discrepancies between the two calculations arise from the differences in target wave functions because of the strong configuration mixing.

Finally, in Figs. 8, 9 we compare the effective collision strengths as a function of electron temperature for the forbidden and dipole-allowed transitions with the R-matrix calculations of Ramsbottom \& Bell (1997). The effective collision strengths are calculated by averaging the total collision strengths
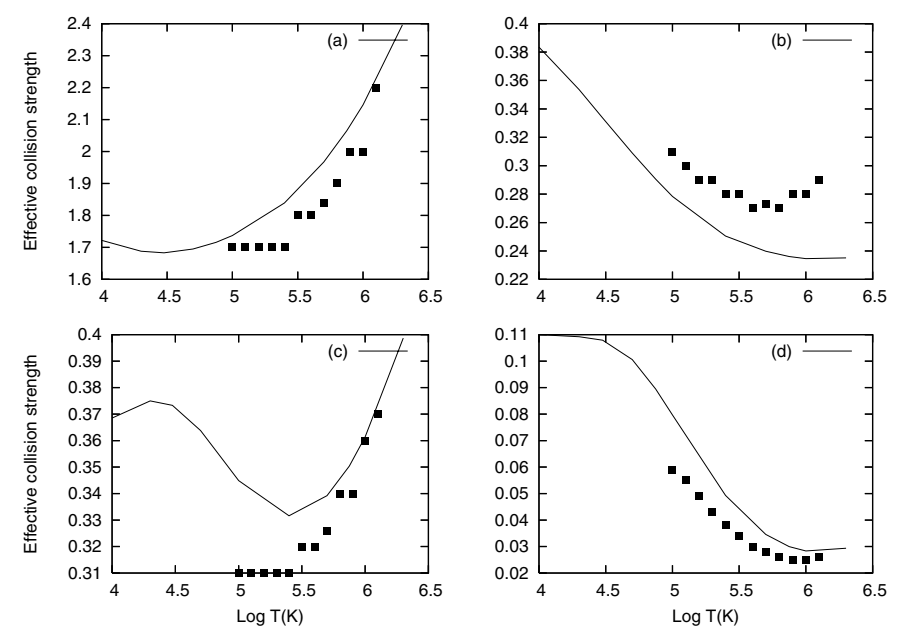

Fig. 9. Effective collision strengths for the dipole-allowed a) $2 s^{2} 2 p^{3}$ ${ }^{4} \mathrm{~S}_{3 / 2}^{\circ}-2 \mathrm{~s} 2 \mathrm{p}^{4}{ }^{4} \mathrm{P}_{5 / 2}(1-6)$, b) $2 \mathrm{~s}^{2} 2 \mathrm{p}^{3}{ }^{2} \mathrm{D}_{3 / 2}^{\circ}-2 \mathrm{~s} 2 \mathrm{p}^{4}{ }^{2} \mathrm{D}_{5 / 2}(3-9)$, c) $2 \mathrm{~s}^{2} 2 \mathrm{p}^{3}$ ${ }^{2} \mathrm{P}_{1 / 2}^{\circ}-2 \mathrm{~s} 2 \mathrm{p}^{4}{ }^{2} \mathrm{D}_{3 / 2}$ (4-10), and d) $2 \mathrm{~s}^{2} 2 \mathrm{p}^{3} \quad{ }^{2} \mathrm{P}_{1 / 2}^{\circ}-2 \mathrm{~s}^{2} 2 \mathrm{p}^{2}\left({ }^{1} \mathrm{D}\right) 3 \mathrm{~s} \quad{ }^{2} \mathrm{D}_{3 / 2}$ (4-22) transitions as a function of electron temperature. Present results, solid curve; Ramsbottom \& Bell (1997), solid rectangles.

over a Maxwellian distribution of electron energies. The effective collision strengths usually exhibit very smooth dependence on temperature despite strong resonance structures in collision strengths. The effective collision strengths for many transitions, however, are significantly enhanced due to presence of resonances at low electron energies. As seen from panels (a), (b), and (d) of Fig. 8, the present effective collision strengths for the $2 \mathrm{~s}^{2} 2 \mathrm{p}^{3}{ }^{4} \mathrm{~S}_{3 / 2}^{\circ}-{ }^{\circ} \mathrm{D}_{5 / 2}^{\circ}(1-2), 2 \mathrm{~s}^{2} 2 \mathrm{p}^{3}{ }^{2} \mathrm{P}_{1 / 2}^{\circ}-{ }^{2} \mathrm{P}_{3 / 2}^{\circ}$ (4-5), and $2 \mathrm{~s}^{2} 2 \mathrm{p}^{3}{ }^{4} \mathrm{~S}_{3 / 2}^{\circ}-2 \mathrm{~s}^{2} 2 \mathrm{p}^{2}\left({ }^{3} \mathrm{P}\right) 3 \mathrm{~s}{ }^{2} \mathrm{P}_{3 / 2}(1-20)$ transitions agree well with the previous R-matrix calculations at all temperatures except low temperatures. This discrepancy at lower temperatures is caused mainly by the differences in total collision strengths in the near-threshold region. There are bigger differences among the two calculations for the $2 \mathrm{~s}^{2} 2 \mathrm{p}^{3}{ }^{4} \mathrm{~S}_{3 / 2}^{\circ}{ }^{2} \mathrm{P}_{1 / 2}^{\circ}(1-4)$, transition at all temperatures as shown in Fig. $8 \mathrm{~b}$. The differences in the background collision strengths and the resonance structures for the forbidden $2 \mathrm{~s}^{2} 2 \mathrm{p}^{3}{ }^{4} \mathrm{~S}_{3 / 2}^{\circ}-{ }^{2} \mathrm{P}_{1 / 2}^{\circ}(1-4)$ transition produce noticeable differences in effective collision strengths. The bigger differences between the calculations are caused by the difference in target wave functions used in two calculations. The effective collision strengths for the dipole-allowed (a) $2 s^{2} 2 p^{3}{ }^{4} S_{3 / 2}^{\circ}-2 s 2 p^{4}$ ${ }^{4} \mathrm{P}_{5 / 2}$ (1-6); (b) $2 \mathrm{~s}^{2} 2 \mathrm{p}^{3}{ }^{2} \mathrm{D}_{3 / 2}^{\circ}-2 \mathrm{~s} 2 \mathrm{p}^{4}{ }^{2} \mathrm{D}_{5 / 2}$ (3-9); (c) $2 \mathrm{~s}^{2} 2 \mathrm{p}^{3}$ ${ }^{2} \mathrm{P}_{1 / 2}^{\circ}-2 \mathrm{~s} 2 \mathrm{p}^{4}{ }^{2} \mathrm{D}_{3 / 2}$ (4-10); and (d) $2 \mathrm{~s}^{2} 2 \mathrm{p}^{3}{ }^{2} \mathrm{P}_{1 / 2}^{\circ}-2 \mathrm{~s}^{2} 2 \mathrm{p}^{2}\left({ }^{1} \mathrm{D}\right) 3 \mathrm{~s}$ ${ }^{2} \mathrm{D}_{3 / 2}$ (4-22) transitions as a function of electron temperature are displayed in Fig. 9 where our results are compared with the previous R-matrix calculation of Ramsbottom \& Bell (1997). Once again there is a reasonable agreement between the two calculations. It may be noted that Ramsbottom \& Bell (1997) gave their results only for two significant digits and a smooth curve could not be obtained in Figs. 8, 9 from their reported results. The differences at lower temperatures are caused by the rich resonance structures with many narrow resonances resolved in our calculation with fine energy grid.

The effective collision strengths between all 74 fine-structure levels of the ground $2 s^{2} 2 p^{3}$ and excited $2 s^{2} p^{4}, 2 p^{5}, 2 s^{2} 2 p^{2} 3 l$, and $2 \mathrm{~s} 2 \mathrm{p}^{3} 3 \mathrm{l}$ configurations are presented in the Table 4 for electron temperatures from 10000 to $200000 \mathrm{~K}$ suitable for astrophysical applications. The indices of the lower and upper levels involved in transitions are given in Table 1 . We have calculated all 


\section{S. S. Tayal: Collision strengths for Mg VI}

partial waves up to $J=30$ in our B-spline Breit-Pauli R-matrix approximation and used a top-up procedure based on geometric series approximation for the forbidden transitions to estimate the contributions from higher partial waves. The top-up procedure based on the Coulomb-Bethe approach was used for the allowed transitions. In order to accurately calculate effective collision strengths at higher temperatures, we also extrapolated our collisions strengths to high energies. The effective collision strengths for the forbidden transitions display a very slow falloff with increasing temperature at higher temperatures. The effective collision strengths for many transitions are significantly enhanced because of the presence of resonances in the total collision strengths at low electron energies.

\section{Summary}

We have presented radiative parameters and effective collision strengths for all transitions among the 74 LSJ levels of Mg VI. This is most extensive and consistent data set for collisional and radiative parameters for $\mathrm{Mg}$ VI to date. The calculations were performed with the BSR code (Zatsarinny 2006) in which a B-spline basis is employed to represent the continuum orbitals. The use of non-orthogonal orbital sets, both constructing the target wave functions and for representing scattering functions, allowed us to optimize different atomic wave functions independently, hence to generate a more accurate description of the target states than those used in previous collision calculations. The radiative parameters for stronger transitions are in good agreement with the recent extensive CI calculations (Froese Fischer \& Tachiev 2004).

The effective collision strengths were presented over a wide range of temperatures suitable for use in astrophysical plasmas modeling. Systematic comparisons with other available calculations were made. The overall agreement with previous R-matrix calculations of Ramsbottom \& Bell (1997) is very reasonable and some discrepancies can be explained on the basis of more accurate target wave functions used in our calculations. Taking the good agreement for oscillator strengths into account, the accuracy for the strong dipole-allowed transitions is estimated to be within $10 \%$. Overall, our results are estimated to be accurate to $\sim 20 \%$ or better for most of the transitions.

Acknowledgements. This research work is supported by NASA grant NNX11AB62G from the Solar and Heliophysics program.

\section{References}

Badnell, N. R. 1999, J. Phys. B, 32, 5583

Bhatia, A. K., \& Landi, E. 2003, At. Data Nucl. Data Tables, 85, 317

Bhatia, A. K., \& Mason, H. E. 1980, MNRAS, 190, 925

Bhatia, A. K., \& Young, P. R. 1998, At. Data Nucl. Data Tables, 68, 219

Brooks, D. H., Warren, H. P., Williams, D. R., \& Watanabe, T. 2009, ApJ, 705, 1522

Burgess, A., \& Sheorey, V. B. 1974, J. Phys. B, 7, 2403

Froese Fischer, C. 2007, Comput. Phys. Commun., 176, 559

Froese Fischer, C., \& Tachiev, G. 2004, At. Data Nucl. Data Tables, 87, 1

Landi, E., \& Bhatia, A. K. 2007, At. Data Nucl. Data Tables, 93, 1

Ramsbottom, C. A., \& Bell, K. L. 1997, A\&AS, 125, 543

Saraph, H. E. 1978, Comput. Phys. Commun., 15, 247

Tayal, S. S., \& Zatsarinny, O. 2008, Phys. Rev. A, 78, 012713

Tayal, S. S., \& Zatsarinny, O. 2010, ApJS, 188, 32

Thomas, R. J., \& Neupert, W. M. 1994, ApJS, 91, 461

Young, P. R., \& Landi, E. 2009, ApJ, 707, 173

Young, P. R., Landi, E., \& Thomas, R. J. 1998, A\&A, 329, 291

Young, P. R., Dupree, A. K., Espey, B. R., \& Kenyon, S. J. 2006, ApJ, 650, 1091 Young, P. R., Zanna, G. D., Mason, H. E., et al. 2007, Publ. Astron. Soc. Japan, 59, 727

Zatsarinny, O. 2006, Comput. Phys. Commun., 174, 273

Zatsarinny, O., \& Froese Fischer, C. 2000, Comput. Phys. Commun., 124, 247

Zatsarinny, O., \& Tayal, S. S. 2001, J. Phys. B, 34, 1299

Zhang, H. L., \& Sampson, D. H. 1999, At. Data Nucl. Data Tables, 72, 153 